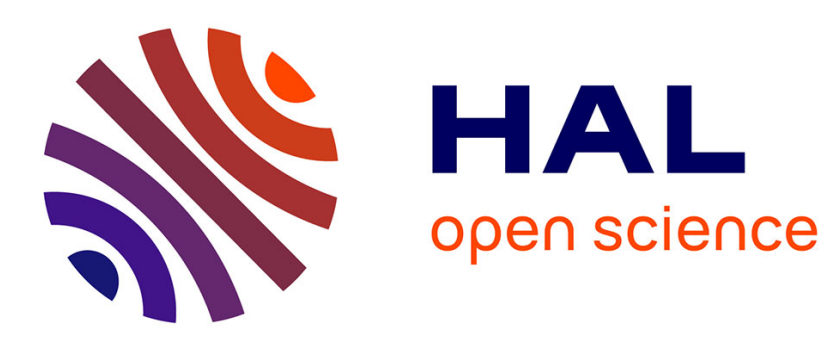

\title{
La réception de la thèse de Lacan par le milieu psychiatrique et ses suites \\ David Monnier
}

\section{To cite this version:}

David Monnier. La réception de la thèse de Lacan par le milieu psychiatrique et ses suites. Annales Médico-Psychologiques, Revue Psychiatrique, 2016, 174 (9), pp.735-739. 10.1016/j.amp.2015.11.014 . hal-01728334

\section{HAL Id: hal-01728334 \\ https://univ-rennes2.hal.science/hal-01728334}

Submitted on 29 Aug 2018

HAL is a multi-disciplinary open access archive for the deposit and dissemination of scientific research documents, whether they are published or not. The documents may come from teaching and research institutions in France or abroad, or from public or private research centers.
L'archive ouverte pluridisciplinaire HAL, est destinée au dépôt et à la diffusion de documents scientifiques de niveau recherche, publiés ou non, émanant des établissements d'enseignement et de recherche français ou étrangers, des laboratoires publics ou privés. 


\section{Mémoire}

\section{La réception de la thèse de Lacan par le milieu psychiatrique et ses suites}

\section{The reception of Lacan's thesis by the psychiatric community and its aftermath}

\section{David Monnier}

Laboratoire de recherches multi-sites EA 4050 " recherches en psychopathologie, nouveaux symptômes et lien social ", 14, place de la Poterie, 56100 Lorient, France

\section{N F O A R T I C L E}

\section{Historique de l'article:}

Reçu le 3 août 2015

Accepté le 8 novembre 2015

Disponible sur Internet le 15 juillet 2016

\section{Mots clés :}

Biographie

Histoire de la psychiatrie

Histoire de la psychanalyse

Lacan Jacques

Relation entre pairs

Travail de fin d'études

Wallon Henri

\section{Keywords:}

Biography

History of psychiatry

History of psychoanalysis

Lacan Jacques

Peer relationship

Wallon Henri

Working graduation

\section{R É S U M É}

Nous avons tenté de déterminer les conditions de passation de la thèse de Lacan. Nous l'avons replacée dans son contexte. Lacan a émis des réserves relatives à l'accueil de sa thèse et sera réticent à sa republication. Il a indiqué avoir fait une erreur à propos de sa thèse. Nous avons envisagé diverses modalités où elle peut résider et ce qu'elle a pu avoir comme implications. Nous avons découvert que le dossier étudiant de Lacan est manquant. Nous avons retrouvé deux comptes rendus méconnus de l'époque, l'un de Lucien Rouquès et l'autre d'Henri Wallon qui a probablement été à l'origine de leurs relations. Nous avons aussi appris incidemment que Lacan a obtenu le prix Baillarger. Lacan n'a pas fait carrière hospitalo-universitaire. Cette période l'aura au moins amené, à travers sa thèse et sa patiente Aimée, à rencontrer la psychanalyse. Il reviendra ensuite sur les apports de certains auteurs pour s'en départir ou les réévaluer. Toutefois, il a toujours eu le souci d'enseigner aux psychiatres, d'une manière ou d'une autre.

(c) 2016 Elsevier Masson SAS. Tous droits réservés.

\section{A B S T R A C T}

Lacan uttered reserves relative to the reception of his thesis and will be reluctant to its republication. Lacan will go back and forth then leave some of the authors who inspired him. However, he always had the concern to teach the psychiatrists, somehow or other.

Objectives. - We shall try to determine the conditions of the doctoral examination of Lacan. We would also try to specify the reception of his thesis, to replace it in its context. We shall aim at finding what Lacan was able to say himself about it on several occasions.

Materials and methods. - Search for references to the thesis of Lacan, confrontation of historical data, analysis and comments of texts. The discussion concerns in particular the academic and medical career of Lacan, his motivation to enter it or his aspiration to step out of it and open up to other circles. So, he indicated having made an error about the thesis. We shall try to envisage the diverse modalities where it can lie and what implications she may have had.

Results. - We discovered that the student file of Lacan is missing. We found two reports underestimated of that time, one of the Lucien Rouquès and other one of Henri Wallon which was probably at the origin of their relations. We also learnt by the way that Lacan obtained the prize Baillarger.

Conclusion. - There are still points to be clarified concerning Lacan and the psychiatry. It however gave fruitful debates. And it has at least brought Lacan, through his thesis and his patient Aimée, to meet psychoanalysis.

(c) 2016 Elsevier Masson SAS. All rights reserved. 


\section{Introduction}

Beaucoup de choses ont été dites sur la thèse de Lacan, De la psychose paranoïaque dans ses rapports avec la personnalité [9], parue en 1932, mais il reste nombre de lacunes quant aux circonstances qui l'entourent ainsi qu'une relative incertitude quant à ses éventuelles répercussions, voire conséquences. Partant de la réticence de Lacan à la republication de sa thèse exprimée en 1975 [11], car il considérait avoir fait là une erreur, nous reviendrons sur l'accueil qui lui a été réservé. Et nous tenterons de dégager quelques éléments indiquant son rapport à la psychiatrie et expliquant qu'il n'y soit pas resté. Nous proposons ainsi une ébauche de reconstitution historique afin de retracer la subjectivité à l'œuvre et la singularité d'un parcours.

\section{Passation. Novembre 1932}

Nous nous sommes intéressé d'abord aux conditions de passation de sa thèse. Nous avons voulu connaître son jury et lire son rapport de soutenance afin de déterminer si un membre du jury avait signalé quelques erreurs ou points de désaccord.

Or, nous avons découvert que, là où il devait être, le dossier étudiant de Lacan a disparu. Il est désormais manquant, alors qu'il n'avait apparemment manqué à personne jusqu'à présent. Dans l'état actuel des choses, on peut pratiquement affirmer qu'il n'y a plus aucune trace administrative disponible de la soutenance de Lacan. On ne peut plus déterminer qui figurait à son jury. Comme me l'a fait remarquer un de mes correspondants, les membres du jury doivent probablement faire partie des remerciements, comme il est de coutume, mais cela laisse encore une liste d'une vingtaine de noms. Sur le document original, il est seulement indiqué le nom du président du jury, Henri Claude, qui était également le directeur de recherche de Lacan, ce qui ne se fait plus guère.

Par ailleurs, la soutenance était la plupart du temps une formalité et durait environ une heure, à l'instar d'une thèse d'exercice en médecine de nos jours. Or, nous apprenons de Lacan lui-même qu'elle ne s'est pas très bien passée. Il relate l'incident suivant :

"Quand je passais ma thèse [...], un de mes maîtres me pria de formuler ce qu'en somme je m'y étais proposé : "En somme, Monsieur, commençai-je, nous ne pouvons oublier que la folie soit un phénomène de la pensée..." Je ne dis pas que j'eusse ainsi suffisamment indiqué mon propos. Le geste qui m'interrompit avait la fermeté d'un rappel à la pudeur : "Ouais ! Et après ? signifiait-il. Passons aux choses sérieuses. Allez-vous donc nous faire des pieds de nez ? Ne déshonorons pas cette heure solennelle. Num dignus eris intrare in nostro docto corpore cum isto voce : pensare !" " [7].

Lacan poursuit : "Je fus nonobstant reçu docteur avec les encouragements qu'il convient d'accorder aux esprits primesautiers. " Comme le système de mentions n'existait pas à l'époque, cette déclaration nous semble à prendre au figuré et à appréhender en opposition aux félicitations. Cela souligne que la soutenance ne s'est pas déroulée dans les conditions idéales. Plus globalement, dans une lettre d'août 1933 à sa maîtresse Olesia Sienkiewicz, Lacan « tempête déjà contre ces années de clinicat qui, disait-il, lui avaient fait perdre un temps précieux » [23]. Cela n'exprime probablement pas son regret d'être devenu psychiatre mais celui de n'être pas venu à la psychanalyse plus tôt.

\section{Comptes rendus, 1932-1933}

La thèse semble avoir été bien accueillie dans certains milieux littéraire, surréaliste et politique [1]. Son originalité tient à la place qu'elle accorde au registre du sens et surtout au langage dans l'approche de la psychose. C'est surtout l'histoire et les écrits
d'Aimée, la patiente principale étudiée par Lacan, qui intéresse notamment Dali, Nizan, Eluard. Ce succès d'estime apporte à Lacan une certaine notoriété.

Dans le milieu médico-psychologique, la thèse obtient deux comptes rendus déjà connus, celui de son ami Henri Ey en 1932 [4] ainsi que celui de Paul Guiraud en 1933 [6]. Nous en avons retrouvé deux, méconnus, voire oubliés, celui de Lucien Rouquès en 1933 [26] mais avant cela celui d'Henri Wallon en 1932 [29]. C'est, selon nous, la première fois que les chemins de Lacan et de Wallon se croisent. Malheureusement, aucun de ces textes sur la thèse n'évoque la passation, le jury ou quelques erreurs qu'aurait pu reconnaître Lacan après-coup.

Ajoutons que Lacan a envoyé un exemplaire de sa thèse à Freud qui en accuse réception poliment sans en faire le moindre commentaire public, a fortiori un compte rendu. Et dans le milieu analytique, la thèse a été à peine prise en considération par la première génération d'analystes.

\section{Le prix Baillarger, mars 1934}

Puis Lacan obtient le prix Baillarger de l'Académie nationale de médecine [27]. Jules Baillarger était un aliéniste du $\mathrm{xIX}^{\mathrm{e}}$ siècle, spécialiste des vésanies sans cause organique. Le prix est officiellement annoncé dans la séance du 13 mars 1934 et décerné le 11 décembre 1934. La dotation de ce prix est de 2500 francs. Pour donner une comparaison [2], une postière débutante à la campagne comme le fut la patiente, Aimée, de Lacan avait un salaire de 750 francs par mois tandis qu'un professeur d'université percevait jusqu'à 7000 francs mensuels. Les attendus du prix sont les suivants : "Ce prix sera décerné à l'auteur du meilleur travail sur la thérapeutique des maladies mentales et sur l'organisation des asiles publics ou privés consacrés aux aliénés. Les mémoires des concurrents devront toujours être divisés en deux parties. Dans la première, ils exposeront, avec observations cliniques à l'appui, les recherches qu'ils auront faites sur un ou plusieurs points de thérapeutique. Dans la seconde, ils étudieront, séparément pour les asiles publics et pour les asiles privés, par quels moyens et au besoin par quels changements dans l'organisation de ces asiles on pourrait faire une part plus large au traitement moral et individuel. »

Ce prix a ainsi couronné par exemple les travaux de Julien Raynier, "L'assistance aux aliénés ", en 1924, ou de Antony Rodiet et Paul Quéron en 1936 sur « La question de l'hérédité des maladies mentales au vingtième siècle ».

Remarquons que la thèse de Lacan ne correspond pas exactement aux attendus de ce prix biennal. Il n'est pas mentionné quels psychiatres ou neurologues de l'Académie ont néanmoins attribué le prix à Lacan mais nous avons retrouvé leur rapport, à défaut de celui de la soutenance : "L'auteur y expose le problème du développement des tendances paranoïaques de certains sujets pour arriver à constituer une véritable maladie autonome qui remanie la personnalité ; c'est la psychose paranoïaque qui s'édifie sur des interprétations, des illusions, et même des hallucinations ; elle aboutit à une activité raisonnante mais primitivement variée en provoquant des conflits vitaux de toutes sortes. L'intérêt de cette forme de psychose est qu'elle aboutit dans un grand nombre de cas à une amélioration ou même à la guérison. L'auteur fait enfin une étude pathogénique très intéressante » [3].

On peut donc estimer que le prix a été justifié, même si Lacan était le seul candidat en lice cette année-là.

\section{Un concours du médicat sans suite et autres perspectives d'avenir, 1934}

À la suite de cela, auréolé de sa gloire naissante, Lacan passe en mai 1934 le concours du médicat destiné au recrutement des médecins chefs des asiles [24]. Cette fois encore, la réussite est 
mitigée dans la mesure où l'un des examinateurs dit « Ce garçon nous ennuie » et que Lacan est reçu onzième sur treize. Or, « malgré son admission, il se déroba à l'obligation de prendre le poste hospitalier qui lui revenait ». On n'en connaît pas la raison exacte mais on peut supposer que ce poste ne l'intéressait pas ou qu'il se trouvait éloigné de Paris. Henri Ey, l'année précédente, avait obtenu un poste à Bonneval (Eure-et-Loir).

Gageons que sa motivation n'était guère engagée, d'autant qu'à la rentrée 1934, il envisage de faire des études de philosophie pour passer l'agrégation de philosophie. Il fréquente alors le milieu philosophique et suit les cours de différents philosophes tels qu'Alexandre Koyré ou Alexandre Kojève. Qui plus est, il pose sa candidature à la Société Psychanalytique de Paris où il est admis comme membre adhérent le 20 novembre 1934.

\section{Après le compte rendu de Wallon, 1936-1938}

Certes, un compte rendu n'implique pas forcément une relation ensuite. Mais le compte rendu de Wallon que nous avons retrouvé est peut-être à l'origine de leur relation dont découlent des élaborations importantes de Lacan. C'est peut-être cela qui a attiré l'attention de Lacan sur les travaux de Wallon relatifs au développement de l'enfant [28]. Lacan s'est inspiré de son "stade du miroir » pour faire une conférence à la Société psychanalytique de Paris le 16 juillet 1936. Elle prépare son intervention au quatorzième congrès de l'Association psychanalytique internationale à Marienbad, en Tchécoslovaquie, le lundi 3 août 1936. C'est la première intervention internationale de Lacan. Il semble qu'elle se soit relativement mal passée, à l'instar de sa soutenance de thèse et du concours du médicat. En effet, Ernest Jones, président de séance et du congrès, a interrompu Lacan car il dépassait les dix minutes imparties. Le texte de Lacan n'a pas été remis au congrès et n'a jamais été publié. L'intervention est seulement signalée comme "The Looking-glass Phase " [18]. Le 17 juillet 1949, Lacan refera une intervention sur ce même thème au seizième congrès de l'Association psychanalytique internationale à Zurich, en Suisse, qui n'aura guère plus d'écho.

Puis en 1938, Henri Wallon demandera à Lacan un article sur les complexes familiaux pour l'Encyclopédie française [13].

\section{Une remarquable absence de candidature à un poste universitaire, 1939-1946}

Par ailleurs, fort de sa thèse, Lacan ne semble pas avoir envisagé de passer le concours d'agrégation. Pourtant, il dit plus tard qu'il a " postulé dans les sphères consacrées de l'ignorance enseignante ", apparemment en le regrettant [17]. On a essayé d'établir si c'est l'agrégation qu'il évoque là, mais en vain car l'administration ne conserve pas la liste des candidats. Son engagement dans la psychanalyse explique en partie qu'il n'ait pas conçu ce projet durant cette période troublée. On peut toutefois souligner qu'il côtoyait alors nombre d'acteurs de la vie universitaire et que certains sont même directement liés à sa carrière hospitalière.

Ainsi, en 1939, lorsqu'il s'agit de remplacer son professeur Henri Claude à la chaire de Clinique des maladies mentales, LaignelLavastine fut choisi. Il était depuis 1931 professeur à la chaire d'Histoire de la médecine. Il chargea alors Georges Parcheminey de réorganiser le département de psychanalyse de l'hôpital SainteAnne. On peut faire l'hypothèse que c'est par ce biais que Lacan obtint un poste à Sainte-Anne.

Puis, en 1942, Laignel-Lavastine est " mis à la retraite ". Joseph Lévy-Valensi est nommé par arrêté du 30 septembre 1942 pour lui succéder. Lacan avait travaillé avec lui dès son internat et l'avait cité dans les dédicaces de sa thèse. Surtout, il a écrit deux articles avec lui, un en 1928 : «Roman policier » [19] et un en 1931 : «Écrits inspirés : schizographie " paru ici même [21]. Il est écrit après le résumé d'une présentation de malade [22] également publié ailleurs [20]. La patiente n'est pas Aimée mais elle fait probablement partie des patientes que Lacan dit avoir étudiées pour sa thèse. L'article va particulièrement dans le sens de la thèse ultérieure de Lacan.

Or, Lévy-Valensi ne prend pas ses fonctions, les lois de Vichy interdisant l'enseignement aux juifs. Il part en zone libre et se réfugie à Nice. Il professe à Aix et à Marseille dans le service d'Henri Roger. Puis, en 1943, il est pris dans une rafle de l'armée allemande et déporté le 20 novembre au camp d'Auschwitz où il meurt trois jours plus tard [5].

Alors, fin 1942, Jean Delay est chargé de ce service à titre intérimaire. Il avait passé sa thèse de médecine en 1935 puis l'agrégation en 1939. Suite à la libération du camp d'Auschwitz par l'Armée rouge, la mort de Lévy-Valensi est attestée. Delay est nommé titulaire de la chaire le $1^{\mathrm{er}}$ janvier 1946. Il était parallèlement chef de service à Sainte-Anne où travaille Lacan et leur relation perdurera bien après cela, sous différentes formes.

\section{Le retour à la thèse, 1946}

En 1946, Lacan est invité à un colloque organisé par Henri Ey à Bonneval. Là, il prend acte publiquement que sa thèse n'a pas eu les échos qu'il espérait. Il revient sur sa thèse en considérant pratiquement qu'il reprend les choses où elles ont été laissées en 1932. Il précisera ultérieurement qu'il a fallu dix ans avant qu'on tienne compte de sa thèse [16]. Il ne fait pas allusion aux grands pontes parisiens mais sans doute plutôt à Tosquelles. Ce dernier est arrivé à l'hôpital de Saint-Alban en 1940 avec la thèse de Lacan en poche. Il l'a mise à l'ordre du jour et s'en est inspiré pour fonder les principes de la psychothérapie institutionnelle et organiser la survie à la campagne durant la guerre.

\section{Les remerciements, au sens négatif, 1946-1966}

Corollairement, Lacan efface d'une certaine manière presque tous les remerciements de sa thèse. Comme s'il voulait ne plus avoir affaire à eux, estimant ne plus rien leur devoir, sinon être quitte. Désormais, il les remercie au sens de leur donner congé. Précisément, il déclare ne plus reconnaître que deux maîtres, Trénel et Clérambault. Son ami Marc Trénel figure dans les remerciements de la thèse à titre posthume. Il était chef du service de l'asile de Maison-Blanche, élève de Paul Sérieux et spécialiste de la psychiatrie légale. Lacan a appris auprès de lui la clinique des troubles du langage en 1928-1929. À la même période, Lacan était dans le service de Clérambault qu'il a ensuite quitté pour suivre Henri Claude et consorts. De sorte que Clérambault était le grand absent des remerciements de sa thèse. Mais Lacan réexaminera son apport en 1956 [14] et le réaffirmera comme son " seul maître dans l'observation des malades " en 1966 [12]. Là, il ne sera radicalement plus question des autres protagonistes de sa thèse.

\section{La réticence à publier, 1975}

Enfin en 1975, à l'occasion de la dite republication de la thèse, Lacan écrit sur la quatrième de couverture : "Thèse publiée (sic) non sans réticence. " Il l'a promis au Seuil ; mais pourquoi et en échange de quoi ? De plus, republier est un bien grand mot dans la mesure où c'est largement une première. Car lors de la première parution, en 1932, il n'y avait guère de distinction entre un imprimeur et un éditeur spécialisé, en l'occurrence Le François. Le 5 septembre 1932, Lacan achève la rédaction de sa thèse que son amie Olga tape. Il apporte ensuite son texte chez l'imprimeur qui donne le bon à tirer le $1^{\mathrm{er}}$ octobre. Il façonne les exemplaires relatifs 
à la soutenance qui a lieu en novembre. C'est la cinq cent trentième de l'année sur six cent quarante. L'usage est d'en tirer quelques exemplaires supplémentaires avec une couverture différente pour le commerce. Nous n'avons pas réussi à déterminer quel en fut le tirage. La librairie de l'éditeur est alors le principal point de vente.

Il semble que Lacan avait déjà essayé de limiter la diffusion de sa thèse en rachetant en 1949 les éventuels exemplaires restants. Si c'est vrai, cela indique qu'il restait des exemplaires disponibles, autrement dit qu'elle n'était alors pas épuisée. On a pu faire l'hypothèse que c'était parce que Lacan venait de découvrir que Didier Anzieu, le fils de sa patiente Aimée, était un de ses analysants depuis janvier 1949. Il n'aurait alors pas souhaité qu'il lise sa thèse où il est éminemment question de lui. Mais une telle démarche nous semble improbable, du moins vaine. Il suffisait à Didier Anzieu, qui préparait alors une thèse sur l'auto-analyse de Freud sous la direction de Daniel Lagache, de se rendre à la bibliothèque de la faculté de médecine, située à quelques pas de la librairie Le François, pour avoir accès à la thèse de Lacan. Ce qu'il fit [25].

Toujours est-il qu'en 1975, la thèse de Lacan n'est effectivement plus disponible dans le commerce. Il ne circule en provenance des ateliers de Saint-Alban qu'un tirage ronéotypé non autorisé. C'est généralement la meilleure méthode pour susciter le désir et inciter à la transgression, ce qui a pu être pratiqué par la suite à l'endroit de ses séminaires.

\section{L'erreur en question, 1975}

C'est alors que Lacan en profite pour reconnaître son erreur, en rédigeant la quatrième de couverture :

- " thèse publiée non sans réticence ;

- à prétexter que l'enseignement passe par le détour de midire la vérité ;

- y ajoutant : à condition que l'erreur rectifiée, ceci démontre le nécessaire de son détour ;

- que ce texte ne l'impose pas, justifierait la réticence » [11].

On ne peut déterminer exactement à quelle erreur il fait allusion parmi celles qu'on a pu envisager ou d'autres. Sans doute pas celle qui n'en est pas vraiment une, d'avoir isolé une forme de paranoïa dont la dénomination a fait long feu. Peut-être celle d'avoir suivi un temps Henri Claude, erreur rectifiée. Assurément pas d'avoir délaissé la médecine ou négligé une carrière universitaire, détours non nécessaires pour la psychanalyse.

En tout cas, cette profession de foi de Lacan ne laisse pas d'évoquer l'amour du chercheur passant par l'erreur plutôt que par le censeur. Ainsi propose-t-on en guise de fin mot de l'histoire de rapprocher cela de la remarque de Lacan disant que ce qu'on attend d'une thèse, c'est "qu'on s'offre à la contradiction " [7]. Propos calqué sur sa formule de l'amour comme "demande que tu me refuses ce que je t'offre » [15]. Gageons que ce qu'il voulait, ce n'était pas ça. Là, à bien des égards, il y a eu ratage à son endroit. Comme chez sa patiente Aimée où il situe désormais une "faillite en ce qui concerne l'accomplissement de l'amour » [8]. Précisément, Lacan reprend cette année-là l'idée développée dans sa thèse : l'échec d'Aimée à " accomplir de façon achevée sa destinée de femme " [10]. Ainsi, sa thèse est restée longtemps en suspens, infinie, ne cessant pas de s'écrire, parfois prisée, parfois méprisée, souvent reprise et reprisée. Alors qu'il n'en attendait probablement pas indifférence butée ou allégeance bornée mais un dépassement dialectique de soi, comme dans l'amour. Ça a toutefois fini par lui arriver.

\section{Conclusion}

La thèse n'est certes pas une réussite objective puisqu'elle n'a pas vraiment prêté à conséquences professionnelles dans le milieu médical. Les relations difficiles de Lacan et la médecine, manifestes à l'occasion de la thèse, entérinent que la médecine était une impasse pour lui.

Il subsiste de nombreuses interrogations et de points à préciser. Toutefois on a entrevu une raison de l'échec subjectif qui a pu être lié à la thèse de Lacan. La recherche implique a minima de trouver la bonne adresse susceptible d'accueillir une thèse. L'erreur là ne fut ni errance ni égarement mais plutôt " erre-ment " dans la mesure où elle a fait émerger une vérité. Lacan s'en est quand même aperçu à temps, relativement rapidement, au moment de conclure sa thèse. Tout ratage comporte sa réussite. Il n'a sans doute pas souhaité faire carrière. L'impétrant ne s'est effectivement pas empêtré à creuser cette veine qui lui apparaissait d'autant plus vaine et bouchée qu'il avait trouvé une issue ailleurs. Il n'aura cependant de cesse de débattre avec les psychiatres et de les inviter à participer à son enseignement.

La réussite de l'interne en médecine fut de passer à autre chose à travers la thèse qui lui a finalement procuré un nouvel horizon. En l'absence de répondant, dans l'attente de quelqu'un à qui parler, il se tourna vers la psychanalyse. Il est entré en psychiatrie pour prendre la porte de la psychanalyse. Sa thèse lui a ouvert une voie princière vers la psychanalyse. En effet, il reste que c'est après la rencontre avec son Aimée et précisément à partir de la rédaction de sa thèse en juin 1932 qu'il commence une analyse qui changea probablement sa perspective ainsi que celle d'une partie du milieu psychiatrique.

\section{Déclaration de liens d'intérêts}

L'auteur déclare ne pas avoir de liens d'intérêts.

\section{Remerciements}

Aux participants à l'exceptionnel colloque " Hors gradus " du 24 juin 2015 à l'Université de Rennes 2 qui a vérifié que la bienveillance va toujours avec la générosité et l'intelligence. L'idée de l'article a germé grâce aux échanges fructueux et chaleureux notamment avec les étudiantes très mobilisées pour cette rencontre et son après-coup.

Aux correspondants qui m'ont aidé à établir les différentes données et informations historiques. Philippe Albou, secrétaire de la Société française d'histoire de la médecine, Céline Benz de la Bibliothèque médicale Henri Ey de Sainte-Anne, Stéphanie Charreaux, Estelle Lambert de la Bibliothèque Inter-universitaire santé, Nathalie Pujol des Archives de la faculté de médecine, Solange Roussier des Archives nationales.

Aux interlocuteurs qui m'ont apporté divers éléments pour la constitution de l'article. Particulièrement Michel Caire, créateur du site Histoire de la psychiatrie, notamment pour ce qui entoure la soutenance de Lacan, et Jérôme van Wijland, directeur de la bibliothèque de l'Académie nationale de médecine notamment pour ce qui concerne le prix Baillarger.

\section{Références}

[1] Allouch J. Marguerite, ou l'aimée de Lacan. Paris: Epel; 1990.

[2] Annuaire statistique de la France. Résumé rétrospectif 1966. Paris: INSEE; 1966.

[3] Brouardel G. Rapport général sur les prix décernés par l’Académie en 1934. Bull Acad Natl Med 1934;112:690.

[4] Ey H. Compte rendu de la thèse de J. Lacan « De la psychose paranoïaque dans ses rapports avec la personnalité ». Encéphale 1932:2:851-6.

[5] Garrabé J, Peneau E. De la schizographie dans les écrits de Joseph Lévy-Valensi. Ann Med Psychol 2014;172:376-81. 
[6] Guiraud P. Analyses. De la psychose paranoïaque dans ses rapports avec la personnalité, par Jacques LACAN. (thèse Paris, 1932, 382 p. Le François éditeur). Ann Med Psychol 1933;91:230-1.

[7] Lacan J. Conclusions du congrès de la Grande-Motte, 4 novembre 1973. Lettres de l'École Freudienne de Paris: 1975;235-44.

[8] Lacan J. Conférence à Yale, 24 novembre 1975. Scilicet 1976;6/7:7-31.

[9] Lacan J. De la psychose paranoïaque dans ses rapports avec la personnalité. Paris: Le François; 1932 [Réédition Paris : Le Seuil, 1975]

[10] Lacan J. De la psychose paranoïaque dans ses rapports avec la personnalité; $1932 ; 263$.

[11] Lacan J. De la psychose paranoïaque dans ses rapports avec la personnalité, 1932, Quatrième de couverture: 1975.

[12] Lacan J. De nos antécédents. Écrits. Paris: Le Seuil; 1966.

[13] Lacan J. Le complexe, facteur concret de la psychologie familiale. Encyclopédie française, 8. Paris: Navarin; 1938 [Réédition, Les complexes familiaux, 1984].

[14] Lacan J. Les psychoses, séminaire III, 1955-1956. Paris: Le Seuil; 1981.

[15] Lacan J. Ou pire, séminaire XIX, 1971-1972. Paris: Le Seuil; 2011.

[16] Lacan J. Propos sur la causalité psychique.In: Journées psychiatriques; Evol Psychiatr 1947;I:123-65 [Réédition. Écrits. Paris : Le Seuil, 1966].

[17] Lacan J. Propos sur la causalité psychique. pp. 168.

[18] Lacan J. The looking-glass phase. Int J Psychoanal 1937;18(1):78.

[19] Lévy-Valensi J, Lacan J, Meignant P. Roman policier. Du délire type hallucinatoire chronique au délire d'imagination. Encéphale 1928;5:550-1.
[20] Lévy-Valensi J, Lacan J, Migault P. Délire et écrits à type paranoïde chez une malade à présentation paranoïde. Encéphale 1931;10:821.

[21] Lévy-Valensi J, Lacan J, Migault P. Écrits inspirés : schizographie. Ann Med Psychol 1931;89:508-22.

[22] Lévy-Valensi J, Lacan J, Migault P. Troubles du langage écrit chez une paranoïaque présentant des éléments délirants du type paranoïde (schizographie). Ann Med Psychol 1931;89:407-8.

[23] Roudinesco E, Lacan J. Esquisse d'une vie, histoire d'un système de pensée. Paris: Fayard; 1993. p. 88

[24] Roudinesco E, Lacan J. Esquisse d'une vie, histoire d'un système de pensée; $1993 ; 114$.

[25] Roudinesco E. pp. 256.

[26] Rouquès J. Revue des thèses. Thèses de Paris. Jacques Lacan. De la psychose paranoïaque dans ses rapports avec la personnalité (Le François, éditeur), Paris 1932. Press Med 1933;19:378.

[27] Van Wiljand J. Inventaire en cours de constitution à partir d'une plaquette éditée par l'Académie nationale de médecine à usage interne; 2015.

[28] Wallon H. Les origines du caractère chez l'enfant. Les préludes du sentiment de personnalité. Paris: Boisvin; 1934 [Réédition Paris : PUF, 2002].

[29] Wallon H. Analyses bibliographiques. Obsessions. Psychasthénies. Délires. États paranoïaques. Syndromes hallucinatoires. 725. J. Lacan. - De la psychose paranoïaque dans ses rapports avec la personnalité. - Un vol. de XIII-381 p. (sic), Paris, Le François, 1933. Ann Psychol 1932;33:479-80. 